\title{
Genomic insights into the uncultivated marine Zetaproteobacteria at Loihi Seamount
}

\author{
Erin K Field ${ }^{1}$, Alexander Sczyrba ${ }^{2}$, Audrey E Lyman ${ }^{3}$, Christopher C Harris ${ }^{1}$, Tanja Woyke ${ }^{4}$, \\ Ramunas Stepanauskas ${ }^{1}$ and David Emerson ${ }^{1}$ \\ ${ }^{1}$ Bigelow Laboratory for Ocean Sciences, East Boothbay, ME, USA; ${ }^{2}$ Center for Biotechnology, Bielefeld \\ University, Bielefeld, Germany; ${ }^{3}$ Department of Biology, Colby College, Waterville, ME, USA and \\ ${ }^{4} U S$ Department of Energy Joint Genome Institute, 2800 Mitchell Drive, Walnut Creek, CA, USA
}

\begin{abstract}
The Zetaproteobacteria are a candidate class of marine iron-oxidizing bacteria that are typically found in high iron environments such as hydrothermal vent sites. As much remains unknown about these organisms due to difficulties in cultivation, single-cell genomics was used to learn more about this elusive group at Loihi Seamount. Comparative genomics of 23 phylogenetically diverse single amplified genomes (SAGs) and two isolates indicate niche specialization among the Zetaproteobacteria may be largely due to oxygen tolerance and nitrogen transformation capabilities. Only Form II ribulose 1,5-bisphosphate carboxylase (RubisCO) genes were found in the SAGs, suggesting that some of the uncultivated Zetaproteobacteria may be adapted to low oxygen and/or high carbon dioxide concentrations. There is also genomic evidence of oxygen-tolerant cytochrome $c$ oxidases and oxidative stress-related genes, indicating that others may be exposed to higher oxygen conditions. The Zetaproteobacteria also have the genomic potential for acquiring nitrogen from numerous sources including ammonium, nitrate, organic compounds, and nitrogen gas. Two types of molybdopterin oxidoreductase genes were found in the SAGs, indicating that those found in the isolates, thought to be involved in iron oxidation, are not consistent among all the Zetaproteobacteria. However, a novel cluster of redox-related genes was found to be conserved in 10 SAGs as well as in the isolates warranting further investigation. These results were used to isolate a novel iron-oxidizing Zetaproteobacteria. Physiological studies and genomic analysis of this isolate were able to support many of the findings from SAG analyses demonstrating the value of these data for designing future enrichment strategies.
\end{abstract}

The ISME Journal (2015) 9, 857-870; doi:10.1038/ismej.2014.183; published online 10 October 2014

\section{Introduction}

Iron is the fourth most abundant element in the Earth's crust and reduced Fe(II) can be oxidized to insoluble Fe(III) oxyhydroxides through abiotic or biotic means. The Zetaproteobacteria, a candidate class of the Proteobacteria phylum, carry out Fe(II) oxidation in marine environments at low temperatures, circumneutral $\mathrm{pH}$ and under microaerophilic conditions. To date, the Zetaproteobacteria have only been identified in habitats with high ferrous iron concentrations. Within this context, they have been detected in a wide range of environments from hydrothermal vent sites, such as Loihi Seamount, where they can dominate the microbial community (Rassa et al., 2009; Emerson and Moyer, 2010; McAllister et al., 2011; Fleming et al., 2013), to coastal environments, where they may be involved

Correspondence: D Emerson, Bigelow Laboratory for Ocean Sciences, 60 Bigelow Drive, East Boothbay, ME 04544, USA.

E-mail: demerson@bigelow.org

Received 3 February 2014; revised 18 August 2014; accepted 25

August 2014; published online 10 October 2014 in biocorrosion of steel structures such as ships and pilings (Dang et al., 2011; McBeth et al., 2011).

The Zetaproteobacteria create distinct extracellular structures, such as helical stalks (Singer et al., 2011) and tubular sheaths (Fleming et al., 2013), that are composed primarily of iron oxyhydroxides and organic polymers (Chan et al., 2011). These structures are a by-product of their metabolism and are capable of creating a structured habitat, both physically and chemically, within the environment. At Loihi Seamount, the focus of this study, these iron mats can be centimeters thick and are greatly affected by opposing gradients of oxygen $\left(\mathrm{O}_{2}\right)$ and $\mathrm{Fe}(\mathrm{II})$ that can result in sharp redoxclines (Glazer and Rouxel, 2009). However, there may also be gradients of other chemical constituents found in the environment that could influence the lifestyle of these organisms such as carbon dioxide $\left(\mathrm{CO}_{2}\right)$, ammonium and hydrogen sulfide (Karl et al., 1988; Garcia et al., 2006; Glazer and Rouxel, 2009). The only current evidence of different populations of Zetaproteobacteria has been from molecular-based studies evaluating the small subunit ribosomal gene, which have suggested that there is a significant 
amount of phylogenetic diversity within the Zetaproteobacteria both globally and at Loihi Seamount (McAllister et al., 2011). Currently available Zetaproteobacteria laboratory isolates represent only a small subset of this diversity as the 16S rRNA gene of these isolates are $99 \%$ similar to each other. Unfortunately, the Zetaproteobacteria remain difficult to grow in the lab, thus cultivationindependent methods, such as single-cell genomics, are currently the best approach to learn more about this diverse yet elusive group.

What is known about the Zetaproteobacteria in regards to their metabolic capabilities and role in the environment has come largely from studying cultures in the laboratory. The cultivated representatives are all closely related to Mariprofundus ferrooxydans strain PV-1, an obligate iron-oxidizing, chemolithoautotroph. Of these, only the closely related strains PV-1 and $M$. ferrooxydans M34 have had their genomes sequenced. Although the laboratory isolates have shed light on some of their metabolic capabilities, the question remains whether the broader phylogenetic diversity within this class is reflective of its metabolic diversity and potential niche specialization. To address this question, genomic data from 23 phylogenetically diverse, uncultivated Zetaproteobacteria genomes from Loihi Seamount were obtained. Comparative genomics was conducted to assess their metabolic capabilities that may lead to niche specialization and compare them with the cultivated representatives, both of which were isolated from Loihi Seamount. This information can provide a better understanding of the ecologic and metabolic role the Zetaproteobacteria may have in this environment.

\section{Materials and methods}

Site description and sample collection

Samples were collected from Loihi Seamount using a custom-built, manually operated syringe-based biomat sampler as described by Fleming et al. (2013). The sampler allowed for accurate collection of the surface layer (top $\mathrm{cm}$ ) of the microbial mat, which can be many centimeters thick. Surface layer iron mat samples from three distinct sites at Loihi Seamount were collected and used for single-cell sorting from Hiolo North Marker 31 (AB-133, AB-137), Hiolo North Marker 39 (AB-602) and Pohaku Marker 57 (AB-604). Two additional bulk mat samples from Hiolo North Marker 39 (AC-673) and Pohaku Marker 57 (AC-675) were collected with a suction sampler collecting approximately the top $3-4 \mathrm{~cm}$ of the mat and were used for single-cell sorting. Upon return to the ship, all samples were immediately preserved in glycerol and stored at $-80^{\circ} \mathrm{C}$ until single-cell sorting. Microscopy of corresponding samples not prepared for single-cell sorting revealed biologically produced Fe(III) oxyhydroxides (i.e. stalks and sheaths) indicative of biologic iron oxidation by Zetaproteobacteria in these samples. Additional site and sample details can be found in Supplementary Table S1 and Fleming et al. (2013).

\section{Single-cell sorting and amplification}

The semiconsolidated mat samples were pretreated before single-cell sorting using fluorescenceactivated cell sorting. Each sample was aspirated with a syringe and needle to disrupt the sample and then diluted 1:10 in filter-sterilized artificial seawater before analysis. Single-cell sorting and amplification has been described previously (Stepanauskas and Sieracki, 2007; Swan et al., 2011, 2013; Woyke et al., 2011). Additional details can be found in Supplementary Information.

Whole genome sequencing, assembly, and quality control Based on their identity and phylogenetic diversity, 23 unique Zetaproteobacteria SAGs (of the total 214 Zetaproteobacteria SAGs identified) were chosen for whole genome sequencing. Eight SAGs were sequenced and assembled through the Bigelow Laboratory Single Cell Genomics Center pipeline and 14 SAGs were sequenced and assembled at the Joint Genome Institute (Walnut Creek, CA, USA) (Supplementary Table S2 and Supplementary Methods). One additional SAG (AB-137-C09) was sequenced twice, once at each location, to confirm sequencing and assembly methods between locations had no effect on the resulting genomes. The sequencing at Joint Genome Institute was part of a Community Sequencing Project and followed the Joint Genome Institute's single-cell minimal draft protocol. Sequencing and assembly through the Single Cell Genomics Center pipeline followed the protocols previously described in Wilkins et al. (2014). Detailed methods for sequencing, assembly and thorough quality control, including contaminant identification and removal, can be found in Supplementary Information. Statistics of the final assemblies can be found in Supplementary Table S2.

\section{Genome completeness estimates}

Genome completeness estimates were determined by calculating the number of conserved copy genes recovered in each genome (Woyke et al., 2009; Garcia et al., 2012; Kamke et al., 2013; Rinke et al., 2013). A total of 76 conserved single copy genes, identified based on clusters of orthologous group (COG) classifications, were found in $97 \%$ of all finished Proteobacteria genomes $(n=938)$ in the Integrated Microbial Genome database at the time of analysis (21 July 2013). Genome completeness estimates for each genome were calculated based on the percent of these 76 conserved single copy genes recovered in each individual genome. 
Sequence classification and OTU designation

Sequences obtained from single-cell PCR screening of multiple displacement amplification products were classified using the Silva Aligner (Pruesse et al., 2007) and imported into the ARB database (Ludwig et al., 2004). Zetaproteobacteria sequences were passed through MOTHUR (Schloss and Handelsman, 2005) and clustered using a 0.03 cutoff. An operational taxonomic unit (OTU) was defined as a group of sequences with $97 \%$ sequence similarity to each other. These sequences were compared with reference sequences from McAllister et al. (2011) to determine which global OTU these sequences correlated with.

\section{Whole genome comparisons}

Amino-acid identities (AAIs) were calculated for all pairs of SAGs as follows: For each pair of SAGs, reciprocal best BLAST hits (E-value $<1 \mathrm{E}-3$ ) were used to predict orthologous genes between the two genomes using the amino-acid sequences of all predicted genes. The AAI was computed as the mean of all BLAST alignments of orthologous genes. Rows and columns in the heatmap were hierarchically clustered, resulting in the dendrogram shown. Average nucleotide identities (ANIs) were calculated using the BLAST-based algorithm for calculating average nucleotide identity (ANIb) tool in JSpecies (v.1.2.1) (Richter and Rosselló-Móra, 2009). All nucleotide ambiguities in the form of N's were removed before analysis. SAG AB-602-E04 was removed from all analyses except the heatmap because of extremely low genome recovery leading to a clear bias in the results.

\section{Phylogenetic analyses}

A maximum-likelihood phylogenetic tree was created with the 16S rRNA gene sequences obtained from assembled SAGs. In SAGs where the 16S rRNA gene was not recovered, the partially recovered $16 S$ rRNA gene from PCR screening was used. Nucleotide sequences were aligned using the MAFFT aligner (Katoh et al., 2002), and the phylogenetic tree was created in RAxML-MPI (v.2.2.3) (Stamatakis, 2006) using the GTR GAMMA nucleotide substitution model with 100 bootstrap iterations. Molybdopterin oxidoreductase and ribulose 1,5bisphosphate carboxylase protein sequences were aligned using ClustalW (Thompson et al., 1994) and further aligned manually. Maximum-likelihood phylogenetic trees were created in MEGA5 (Kumar et al., 2008) using the Poisson Substitution Model with 1000 bootstrap iterations.

Isolation of Mariprofundus sp. EKF-M39

An iron mat sample was collected during a recent expedition to Loihi in March 2013 from the Marker
39 iron mat (sample: J2-674-BM1-B456). For the initial enrichment, $100 \mu \mathrm{l}$ of mat sample was added to Balch Tubes containing sterile artificial seawater (ASW) (Emerson and Floyd, 2005) prepared under anaerobic conditions with a headspace of argon: $\mathrm{CO}_{2}$ (80:20) mix. Artificial seawater was prepared using equivalent moles of nitrogen in the form of sodium nitrate to replace ammonium chloride, the usual $\mathrm{N}$-source for artificial seawater. ATCC Trace Mineral Supplement (ATCC, Manassas, VA, USA), ATCC Vitamin Supplement (ATCC) and $500 \mu \mathrm{M} \mathrm{FeCl}{ }_{2}$ (final concentration) were also added. Trace $\mathrm{O}_{2}$ was likely present from addition of the vitamins and mat inoculum. Cultures were transferred under the same growth conditions five times to obtain a pure culture, which was confirmed by microscopy and $16 \mathrm{~S}$ rRNA gene sequencing.

\section{Accession numbers}

The small subunit genes obtained from multiple displacement amplification products have been submitted to GenBank under accession numbers (KM042429-KM042846). Isolate genome scaffolds can be found in GenBank under Mariprofundus ferrooxydans PV-1 (AATS00000000.1) and M. ferrooxydans M34 (NZ_ARAU00000000.1), as well as in Integrated Microbial Genome database under Taxon IDs 639857004 and 2513237158, respectively. Mariprofundus sp. EKF-M39 and all single amplified genomes (SAGs) have been made public in the Integrated Microbial Genome database. Strain EKF-M39 is provided under Taxon ID 2571042360, and SAG Taxon IDs are reported in Table 1.

\section{Results}

Zetaproteobacteria community structure

Microbial community composition determined by $16 \mathrm{~S}$ rRNA gene sequencing of SAGs was in agreement with previous studies of the Loihi Seamount mats (McAllister et al., 2011; Fleming et al., 2013). The Zetaproteobacteria dominated all SAG bacterial $16 S$ rRNA gene libraries $(52-71 \%$ total SAGs) except for the bulk mat sample collected from Marker 57 (Supplementary Figures S1 and S2 and Supplementary Table S3). A total of 214 SAGs were identified as Zetaproteobacteria. These SAGs represented 5 of the 12 Zetaproteobacteria OTUs at Loihi Seamount previously reported by McAllister et al. (2011) including the globally ubiquitous OTUs 1 and 2 (Figure 1 and Supplementary Table S4). Interestingly, no $16 \mathrm{~S}$ sequences from OTU 11 were recovered, indicating that none of the SAGs were closely related to strains $M$. ferrooxydans PV-1 and M34. Marker 57 samples were largely comprised of OTUs 4 and 10 members, whereas Marker 31 and Marker 39 samples were dominated by OTU 1 and 2 members, which is consistent with geographical 
Table 1 Zetaproteobacteria SAG general statistics

\begin{tabular}{|c|c|c|c|c|c|c|c|c|c|c|c|}
\hline$S A G I D$ & $\begin{array}{l}\text { Collection } \\
\text { site }\end{array}$ & $\begin{array}{c}\text { OTU } \\
\text { designation }\end{array}$ & $\begin{array}{c}\text { IMG Taxon } \\
I D\end{array}$ & $\begin{array}{l}\text { Size } \\
(M b)\end{array}$ & $\begin{array}{l}\text { Estimated } \\
\text { genome } \\
\text { recovery (\%) }\end{array}$ & $\begin{array}{c}G C \\
\text { content } \\
(\%)\end{array}$ & $\begin{array}{l}\text { No. of } \\
\text { predicted } \\
\text { genes }\end{array}$ & $\begin{array}{l}\text { No. of protein- } \\
\text { coding genes }\end{array}$ & $\begin{array}{l}\text { No. of } \\
\text { RNAS }\end{array}$ & $\begin{array}{l}\text { No. of } \\
\text { tRNAs }\end{array}$ & $\begin{array}{l}\text { No. of protein- } \\
\text { coding genes } \\
\text { w/functional } \\
\text { prediction }\end{array}$ \\
\hline AB-137-J06 & Marker 31 & 1 & 2264867010 & 0.89 & 47.4 & 46.4 & 1035 & 1021 & 14 & 8 & 776 \\
\hline AB-602-C20 & Marker 39 & 1 & 2264867008 & 0.99 & 21.1 & 46.1 & 1091 & 1072 & 19 & 12 & 795 \\
\hline AB-133_D10 & Marker 31 & 1 & 2524614788 & 1.57 & 54.0 & 47.5 & 1631 & 1605 & 26 & 22 & 1267 \\
\hline AB-137_M18 & Marker 31 & 1 & 2524614790 & 0.45 & $<10$ & 49.4 & 497 & 487 & 10 & 6 & 346 \\
\hline AB-602_L11 & Marker 39 & 1 & 2524614791 & 0.33 & $<10$ & 48.5 & 353 & 345 & 8 & 3 & 272 \\
\hline AB-133-C04 & Marker 31 & 2 & 2264867014 & 1.30 & 73.7 & 43.6 & 1542 & 1514 & 28 & 17 & 1146 \\
\hline AB-137-I08 & Marker 31 & 2 & 2265123003 & 2.05 & 81.6 & 43.2 & 2392 & 2340 & 52 & 39 & 1790 \\
\hline AB-602_F03 & Marker 39 & 2 & 2524614781 & 0.99 & 31.6 & 42.9 & 1085 & 1068 & 17 & 15 & 838 \\
\hline AB-604_P22 & Marker 57 & 2 & 2524614792 & 0.61 & 49.1 & 43.2 & 725 & 716 & 9 & 6 & 557 \\
\hline AB-133_M17 & Marker 31 & 2 & 2524614793 & 1.49 & 77.6 & 42.9 & 1621 & 1589 & 32 & 25 & 1293 \\
\hline AB-137_G16 & Marker 31 & 2 & 2524614794 & 0.89 & 59.2 & 42.9 & 1016 & 995 & 21 & 15 & 768 \\
\hline AB-604-O16 & Marker 57 & 4 & 2264867012 & 0.98 & 46.1 & 50.5 & 1167 & 1141 & 26 & 19 & 896 \\
\hline AB-604_O11 & Marker 57 & 4 & 2524614795 & 0.67 & 26.3 & 48.9 & 764 & 743 & 21 & 10 & 502 \\
\hline AC-673-C02 & Marker 39 & 4 & 2528768162 & 0.88 & 56.6 & 50.3 & 983 & 971 & 12 & 6 & 751 \\
\hline AC-675-C07 & Marker 57 & 4 & 2528768166 & 0.62 & 15.8 & 49.6 & 724 & 691 & 33 & 19 & 435 \\
\hline AB-137-C09 & Marker 31 & 6 & 2264867009 & 2.45 & 82.9 & 47.6 & 2482 & 2432 & 50 & 37 & 1824 \\
\hline AB-137_C09B & Marker 31 & 6 & 2524614796 & 2.35 & 82.9 & 48.1 & 2301 & 2239 & 62 & 44 & 1674 \\
\hline AB-602-E04 & Marker 39 & 6 & 2264867015 & 0.45 & 15.8 & 46.1 & 525 & 517 & 8 & 3 & 371 \\
\hline AB-133-G06 & Marker 31 & 6 & 2264867013 & 1.10 & 56.6 & 46.7 & 1247 & 1222 & 25 & 13 & 925 \\
\hline AB-137-L23 & Marker 31 & 6 & 2528768164 & 1.70 & 73.7 & 47.6 & 1751 & 1710 & 41 & 33 & 1259 \\
\hline AB-604-B04 & Marker 57 & 10 & 2264867011 & 0.95 & 39.5 & 50.2 & 1095 & 1080 & 15 & 11 & 812 \\
\hline AC-673-M07 & Marker 39 & 10 & 2524614605 & 1.48 & 68.4 & 50.9 & 1635 & 1610 & 25 & 20 & 1232 \\
\hline AC-673-N02 & Marker 39 & 10 & 2528768165 & 0.95 & 10.5 & 51.3 & 1094 & 1056 & 38 & 22 & 775 \\
\hline AC-673-B17 & Marker 39 & 10 & 2528768224 & 1.33 & 17.1 & 51.7 & 1458 & 1428 & 30 & 19 & 1076 \\
\hline
\end{tabular}

Abbreviations: GC, Guanine Cytosine; IMG, Integrated Microbial Genome; OTU, operational taxonomic unit; SAG, single amplified genome. ${ }^{a}$ Comparable to OTU designations in McAllister et al. (2011).

differences observed in previous studies at Loihi Seamount (McAllister et al., 2011, Singer et al., 2013). Markers 31 and 39 are geographically close to each other located only a few meters apart at a water depth of $1300 \mathrm{~m}$ near the bottom of Pele's Pit, a crater created as a result of an eruption in 1996. At the time of sampling in 2009, the vent fluid temperature was $\sim 42{ }^{\circ} \mathrm{C}$, while the ambient seawater was around $4{ }^{\circ} \mathrm{C}$. Conversely, Marker 57 is more distant at the Pohaku outcrop ( $400 \mathrm{~m}$ south of Pele's Pit), shallower (water depth of $1178 \mathrm{~m}$ ), has cooler vent fluid temperatures $\left(\sim 28{ }^{\circ} \mathrm{C}\right)$, and a slightly higher $\mathrm{pH}$ (5.6-7.3 compared to 5.8-6.5) (Glazer and Rouxel, 2009). These differences in geographical location, water depth, temperature, and could all have a role in the Zetaproteobacteria community structures observed. The entire summit of Loihi Seamount is considered an $\mathrm{O}_{2}$ minimum zone with seawater $\mathrm{O}_{2}$ concentrations ranging from 50 to $80 \mu \mathrm{M}$ while the vent fluid Fe(II) concentrations are typically several $100 \mu \mathrm{M}$ (Glazer and Rouxel, 2009), indicating that $\mathrm{O}_{2}$ and $\mathrm{Fe}(\mathrm{II})$ are likely not the sole contributors to the differences in community structure as they do not vary significantly between sites. At Marker 39 there were also differences between the surface layer and suction samples of bulk mat, suggesting that OTUs 2 and 6 may be more prevalent in the first centimeter of the mat, while OTUs 1, 4 and 10 may be deeper in the mat. These surface layer samples were collected in consecutive years, thus this is solely an observation, but it

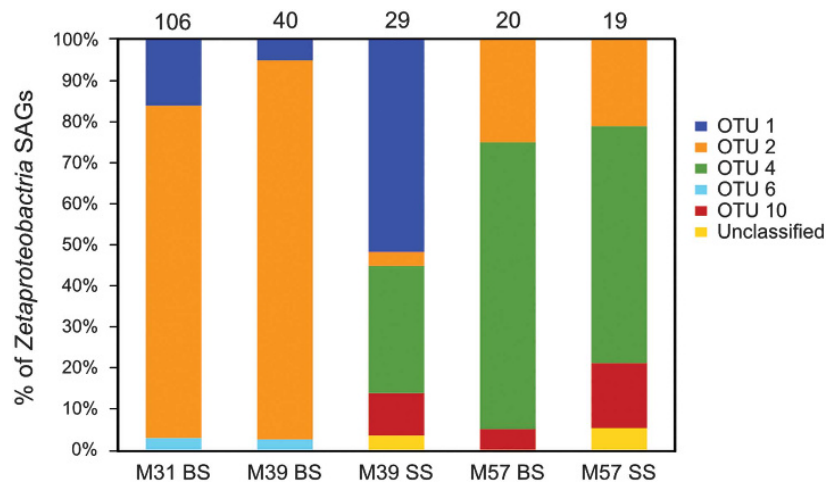

Figure 1 OTU classifications of all Loihi Seamount Zetaproteobacteria 16S rRNA gene sequences identified in each SAG library. Numbers at the top of each bar represent the total number of Zetaproteobacteria SAGs found in that sample and each bar represents a different environmental sample. BS, biomat sample; M31: Marker 31; M39: Marker 39; M57: Marker 57; SS, suction sample.

suggests that there may be some structuring of these organisms with depth in the mat.

Twenty-three SAGs were chosen for sequencing from these three distinct sites within the Loihi Seamount area representing phylogenetically diverse members of the Zetaproteobacteria: OTU 1 (five SAGs), OTU 2 (six SAGs), OTU 4 (four SAGs), OTU 6 (four SAGs) and OTU 10 (four SAGs) (Table 1 and Figure 2) to capture as much diversity as possible. Genome recovery estimates ranged between $<10 \%$ and $83 \%$ with an average of $49 \%$. 


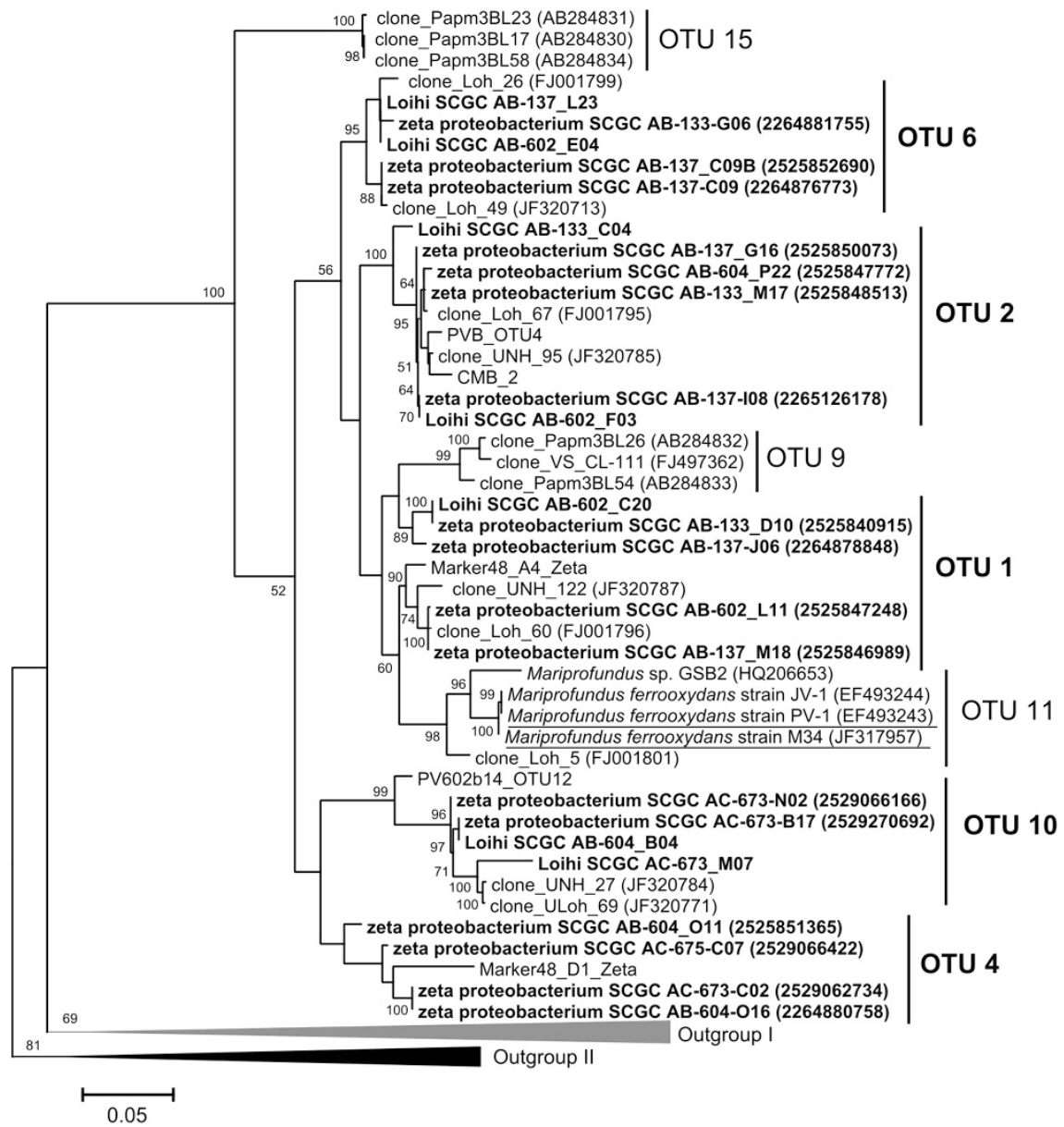

Figure 2 Maximum-likelihood phylogenetic tree of the Zetaproteobacteria SAG 16S rRNA genes. OTUs with representative SAGs are in bold. Sequenced isolates are underlined. Additional Zetaproteobacteria reference sequences were included. The tree was rooted with additional outgroups including other Proteobacteria classes (Outgroup I) and other phyla members (Outgroup II). The tree represents 100 iterations and bootstrap values below 50 are not reported.

General comparisons between Zetaproteobacteria genomes Genomes were compared at both the nucleotide and protein level to determine if phylogenetic similarity correlated with overall genetic similarity. Hierarchical clustering of the genomes based on average AAIs indicated that members of the same OTU did not always cluster together (Figure 3). The OTUs that appear to be most greatly affected were OTUs 1 and 6. There was a general trend in which greater average AAIs correlated with genomes from the same OTU (average 76\%) rather than genomes between OTUs (average 57\%). This trend was consistent regardless of whether AAI or ANI was used (Supplementary Figure S3 and Supplementary Tables S5, S6, and S7), athough outliers were present in both instances. As expected, the number of outliers was higher for ANI than AAI comparisons. These outliers could not be explained by possible cross-contamination or chimeras as the genomes were not sequenced together and quality control measures removed chimeric sequences. It is possible that genome recovery size has a role, but only SAG-602-E04, the smallest genome recovered, is consistent with this. These results also indicate that the isolates are highly similar at the whole genome level, but quite distinct from the SAGs. Although previous studies have suggested that the 16S rRNA gene may not be the best indicator of overall genetic relatedness for many microorganism (Konstantinidis and Tiedje, 2005; Konstantinidis et al., 2006; Staley, 2006; Swan et al., 2013), this is the first evidence that this is also true for the Zetaproteobacteria.

COG comparison analyses suggested that there were no significant metabolic differences when comparing unique COGs in the Zetaproteobacteria genomes between geographic site, mat sample type (i.e. surface layer or bulk mat) and OTU designation. The most significant differences were between the SAGs and isolates, likely due to the phylogenetic breadth of the SAGs compared with the isolates. There were 632 unique COGs found in the SAGs compared with the 124 unique COGs found in the isolates (Supplementary Table S8), which correspond to $30 \%$ and $7 \%$ of the total unique COGs identified in each, respectively. This also indicates that the majority of COGs in both groups are shared. However, protein clustering indicated that proteins were not highly conserved between the 


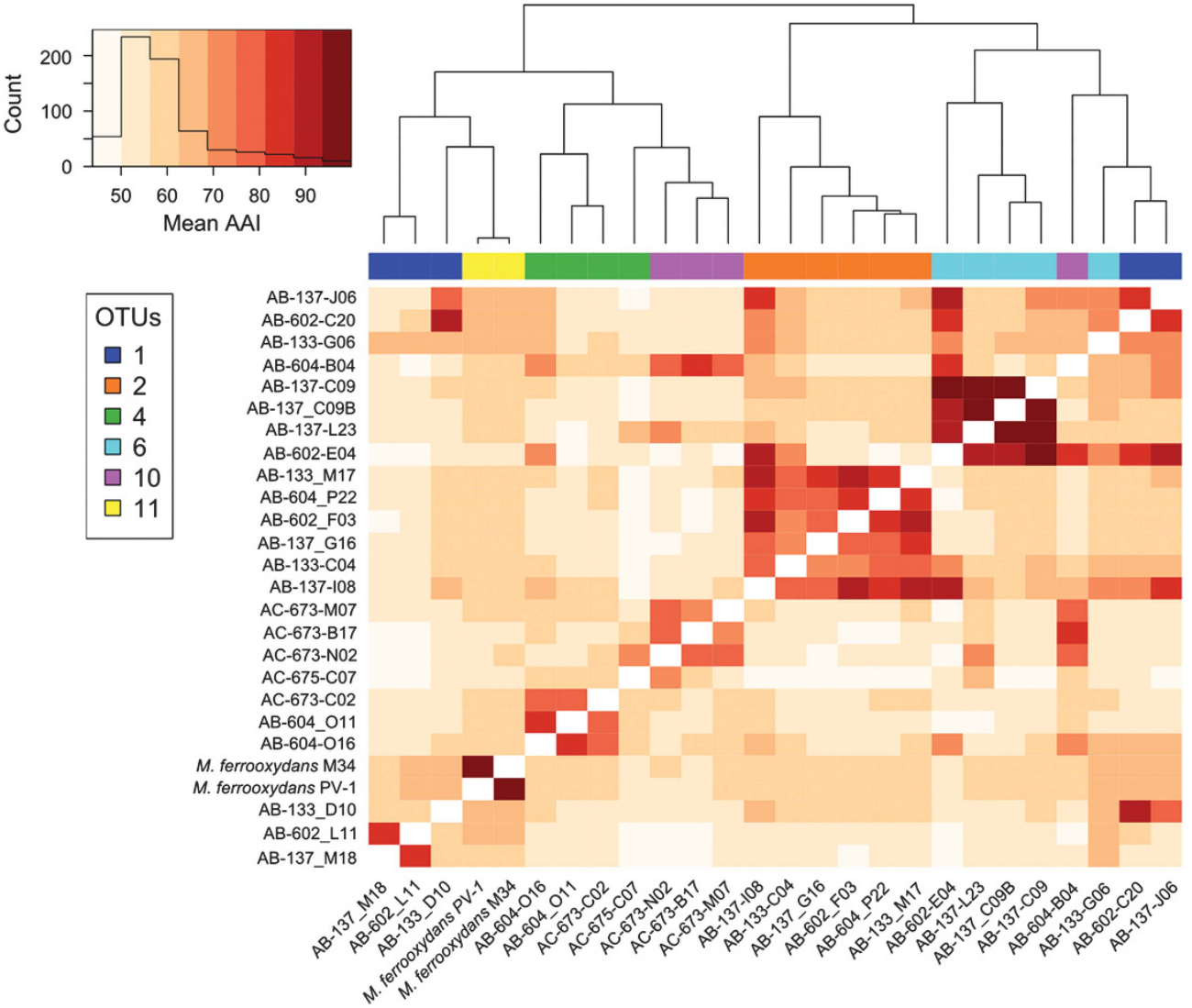

Figure 3 Hierachical clustering heatmap and dendogram of average amino acid identities (AAI) between all Zetaproteobacteria genomes in relation to their designated OTU. Genome self-comparisons are presented in white.

isolates and SAGs, regardless of COG category (Supplementary Figure S4 and Supplementary Table S9). Overall, these results suggest that the genomic variation observed by ANI and AAI comparisons may be due to subtle variations such as environmental responses or evolutionary influences. The subsequent sections will address some of these differences.

\section{Carbon utilization}

Studies of Zetaproteobacteria cultures that were isolated using autotrophic media have indicated that they are obligate autotrophs (Singer et al., 2011). Strains PV-1 and M34 have complete pathways for carrying out carbon fixation using the Calvin-Benson-Bassham cycle. Eleven of the 23 SAGs analyzed here also show evidence of the Calvin-Benson-Bassham cycle, based on the presence of either the ribulose 1,5-bisphosphate carboxylase (RubisCO) gene or the phosphoribulokinase gene, both of which are specific to this pathway (Berg, 2011) (Supplementary Table S10). The detection of these genes did not correlate to genome size or OTU designation. There was no evidence of other carbon fixation mechanisms in any of the Zetaproteobacteria genomes. Both strains PV-1 and M34 contain a Form I and Form II large subunit
RubisCO gene. Large subunit RubisCO genes were recovered from 8 of the 23 SAGs. Three additional SAGs contained RubisCO activation genes $c b b Q$ and $c b b O$. Interestingly, all eight of the RubisCO genes found in the SAGs were of the Form II type (Figure 4). Form II RubisCO genes were notably absent from OTU 4 genomes, but at least one of these genomes had other genes from the Calvin-BensonBassham pathway, suggesting that they may still be capable of autotrophy.

The probability that Form I may have been present but not detected in any of the 23 analyzed SAGs because of incomplete genome recovery is extremely low (estimated 7.49E - 08\%, Supplementary Methods) if it is assumed that multiple displacement amplification biases and subsequent genome coverage is random (Lasken and Stockwell, 2007). Therefore, while both isolates encode Form I and Form II RubisCO genes, it is unlikely that all Zetaproteobacteria do. The presence of Form II rather than Form I is interesting as it is indicative of adaptations to high $\mathrm{CO}_{2}$ and very low $\mathrm{O}_{2}$ environments (Badger and Bek, 2008; Tabita et al., 2008), which is consistent with the $\mathrm{O}_{2}$ profiles presented by Glazer and Rouxel (2009), in which the $\mathrm{O}_{2}$ levels at Loihi Seamount iron mats were often below the detection limit $(3 \mu \mathrm{M})$ even at the mat surface. These findings suggest that the broad 


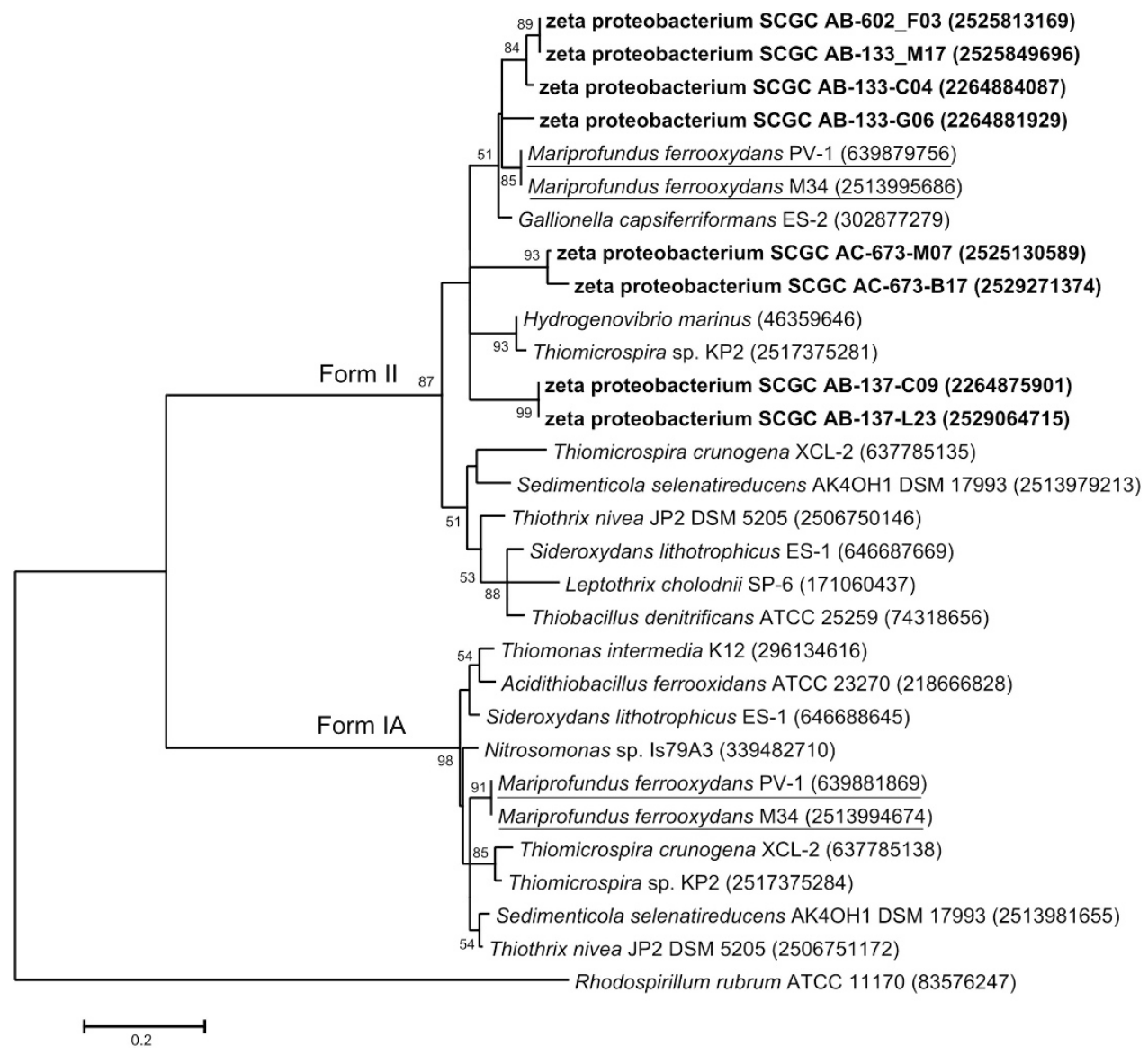

Figure 4 Maximum-likelihood phylogenetic tree of the RubisCO proteins of all Zetaproteobacteria SAGs (bold), M. ferrooxydans PV-1, and M34 (underlined) and other reference organisms. Numbers within parentheses are gene identification numbers. Bootstrap values are representative of 1000 iterations and those values below 50 are not shown.

phylogenetic spectrum of Zetaproteobacteria represented by SAGs analyzed in this study have a lower $\mathrm{O}_{2}$ tolerance for fixing carbon compared with their cultivated counterparts. Decreased $\mathrm{O}_{2}$ and/or increased $\mathrm{CO}_{2}$ may represent favorable conditions for future cultivation efforts.

Nine of the 23 SAGs, as well as strains PV-1 and M34, show the potential for carbohydrate uptake through the phosphotransferase system (Supplementary Table S11). These genes were found across all OTUs with the exception of OTU 4 and include the mannose/fructose-specific component IIA and the Ntr-type mannitol/fructose-specific component IIA genes. As the phosphotransferase system genes may have a complex functional role in the cell (Deutscher et al., 2014), the specific role of these genes in the Zetaproteobacteria remains uncertain. Seven SAGs from three OTUs (1,4 and 10) have endoglucanases, $\beta$-glucosidases, xylanases, and chitin deacetylases, suggesting that there is a genetic potential for degradation and utilization of complex carbohydrates. The presence of these genes suggests that there is a potential for heterotrophy or possibly mixotrophy among some of the Zetaproteobacteria, although there is no evidence to date that either strain PV-1 nor M34 can grow using any carbon source other than $\mathrm{CO}_{2}$.
Potential for iron oxidation

Neither the Zetaproteobacteria SAGs nor isolates contain homologs to well-characterized genes involved in iron oxidation identified in other microorganisms including iro (Amouric et al., 2011), rus (Cox and Boxer, 1978), mtoAB (Shi et al., 2012), pioABC (Jiao and Newman, 2007), foxEYZ (Croal et al., 2007) and cyt579 (Singer et al., 2008). Previous studies have suggested that the Zetaproteobacteria may use a molybdopterin oxidoreductase and alternative respiratory complex III mechanism to carry out iron oxidation (Singer et al., 2011). Molybdopterin oxidoreductase genes were found in 12 of the Zetaproteobacteria SAGs and in strain M34 forming two phylogenetic clusters (Figure 5a). Cluster 1 contains 11 SAG proteins predicted to be nitrate reductases. Cluster 2 contains the molybdopterin oxidoreductase proteins associated with alternative respiratory complex III homologs from strains PV-1 and M34, two SAGs as well as freshwater iron-oxidizing bacteria Gallionella capsiferriformans ES-2 and Sideroxydans lithotrophicus ES-1. The alternative respiratory complex III syntenous operon structure in members of Cluster 2 is conserved and in agreement with previous findings (Singer et al., 2011) (Figure 5b). 

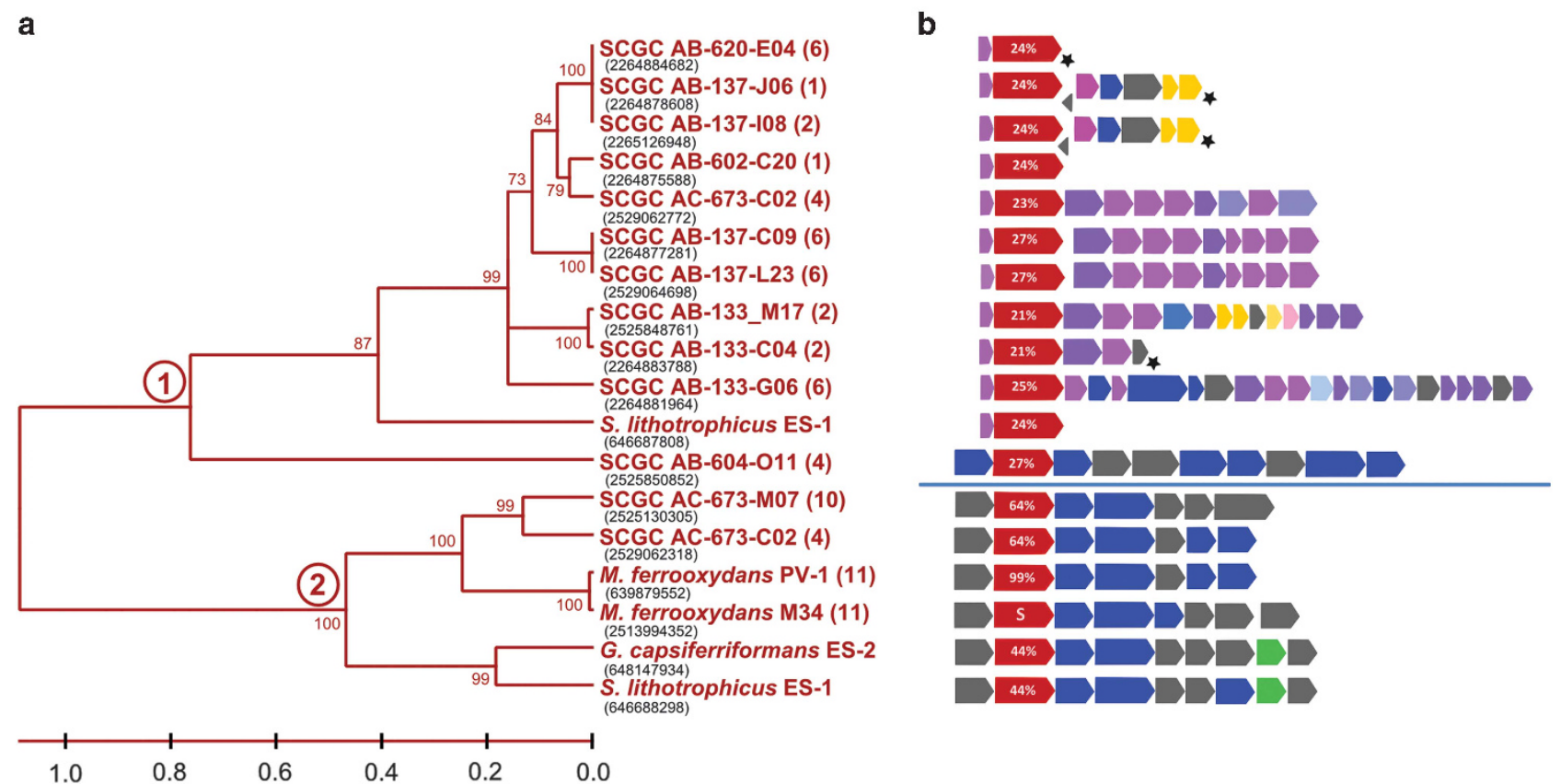

Figure 5 Protein phylogeny (a) and gene operon synteny (b) of the molybdopterin oxidoreductase protein of $M$. ferrooxydans PV-1, M. ferrooxydans M34 and the SAGs with homologs. Molybdopterin oxidoreductase protein (designated in red) phylogeny is presented in the linearized maximum-likelihood phylogenetic tree. BLAST similarities to $M$. ferrooxydans PV-1 (designated with an S) are presented on the gene of interest. OTU designations are listed within parentheses and gene ID numbers are in black. The blue line separates the two distinct clusters identified in the maximum-likelihood tree in (a). The black stars represent the end of the gene operon due to the end of a contig. Gene colors are indicative of COG functional category colors except for the molybdopterin oxidoreductase genes (in red), which correlate with the proteins in (a). Color Code: green, category O; dark blue, category C; light blue, category E; pale blue, category G; dark purple, category H; light purple, category P; violet, category Q; fuchsia, category L; light pink, category J; yellow, category T; light yellow, category D; gray, no category designated.

In contrast, the associated Cluster 1 operon in the SAGs is comprised of genes from different COG categories than those associated with Cluster 2. Many of the genes in Cluster 1's operon are unique to SAGs, with no homologs in either strain PV-1 or M34. These genes include nitrite reductases, molybdenum co-factor biosynthesis genes, molybdenum transporters, molybdopterin-converting factors, and lactate dehydrogenase. SAG AB-604_O11 encodes the only member of Cluster 1 that is a predicted ferredoxin-type nitrate reductase. While this protein is phylogenetically similar to those in Cluster 1 , the gene operon structure is much more similar to Cluster 2's alternative respiratory complex III homologs. In all, these results pose intriguing questions about the functional role and evolution of these molybdopterin oxidoreductase genes and corresponding operons in the Zetaproteobacteria and specifically what role, if any, they may actually have in iron oxidation.

\section{Cytochromes and electron transport}

Strains PV-1 and M34 as well as fourteen SAGs encode a $\mathrm{cbb}_{3}$-type cytochrome $c$ oxidase, which has a high affinity for $\mathrm{O}_{2}$ (Pitcher and Watmough, 2004) and is used under microaerophilic conditions. Notably, $c b b_{3}$ oxidase genes were not recovered from any of the OTU 4 SAGs and only from one of the OTU 10 SAGs (Supplementary Table S12).
Instead, these SAGs have a more $\mathrm{O}_{2}$-tolerant cytochrome $c$ oxidase, suggesting that they are adapted to higher $\mathrm{O}_{2}$ conditions. Strain M34 and eight SAGs, including members of OTU 10, have the bd-I ubiquinol oxidase, which is also used under microaerophilic conditions (Borisov et al., 2011). The presence of both high $\mathrm{O}_{2}$ and low $\mathrm{O}_{2}$ adapted cytochromes suggests that OTU 10 members may be exposed to both low and high $\mathrm{O}_{2}$ conditions. Therefore, this presents intriguing questions regarding the conditions under which they are exposed to higher $\mathrm{O}_{2}$ concentrations, as well as what would constitute a higher $\mathrm{O}_{2}$ concentration. This is also surprising as it was observed that OTU 10 members were more prevalent in the bulk mat samples, suggesting that they may be found deeper within the mats, which is not where higher $\mathrm{O}_{2}$ concentrations would be expected.

There is an operon of $\sim 20$ genes that appears to be highly conserved among the Zetaproteobacteria. Emerson et al. (2013) pointed out this large cluster, composed of two distinct subclusters, is conserved between strain PV-1 and S. lithotrophicus ES-1. Acidophilic iron-oxidizing Leptospirillum spp. also show similarities at the amino-acid level to many of these genes (Emerson et al., 2013). Homologs to these genes were identified in 10 of the SAGs (Figure 6). Interestingly, no homologs were found in any OTU 4 or 10 SAGs. Most of the genes in this 
S. lithotrophicus ES-1 (646690335)

M. ferrooxydans PV-1 (639882347)

SCGC AB-602_F03 (2) (2525812613)

SCGC AB-133_M17 (2) (2525848140)

SCGC AB-137_G16 (2) (2525850176)

SCGC AB-137-I08 (2) (2265127091)

SCGC AB-133_D10 (1) (2525840514)

SCGC AB-137-J06 (1) (2264878575)

SCGC AB-604_P22 (2) (2525847931)

SCGC AB-137-C09 (6)

(2264877792)

SCGC AB-133-C04 (2) (2264883207)

M. ferrooxydans M34 (2513995536)

SCGC AB-133-G06 (6) (2264881601)
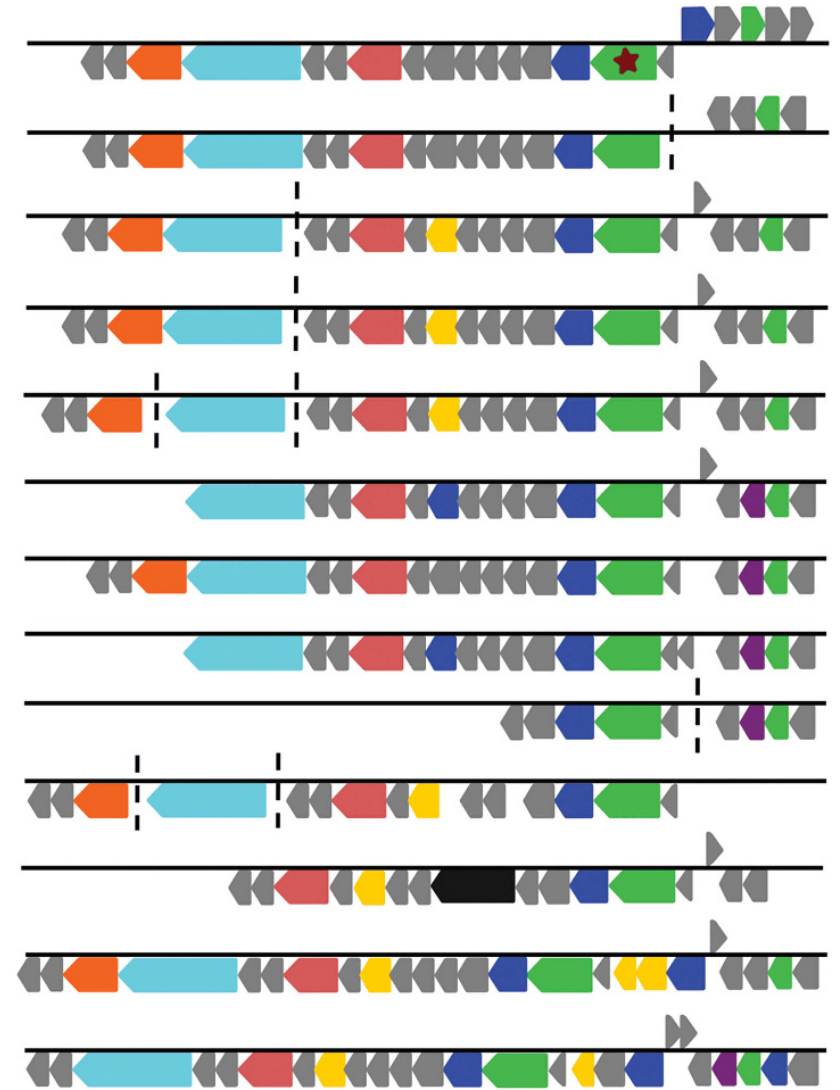

cytochrome c oxidas

subunit I

cytochrome c oxidase subunit II

cytochrome c and c553

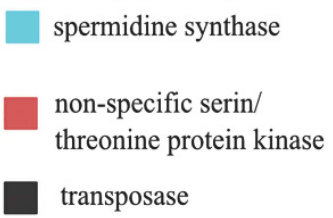

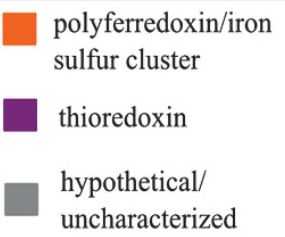

Figure 6 Gene operon synteny of conserved gene cluster homologs between freshwater iron oxidizer S. lithotrophicus ES-1, and Zetaproteobacteria. Gene ID numbers corresponding to homologs of $S$. lithotrophicus cytochrome $c$ oxidase, $c b b_{3}$-type gene (labeled with a star) are provided under genome names. OTU designations are provided within parentheses. Dashed lines designate non-contiguous gene operons and directionality corresponds to that of S. lithotrophicus ES-1.

cluster are hypothetical; however, the few that have annotations indicate that they are likely involved in electron transport. The presence of this large gene cluster in many of the Zetaproteobacteria, S. lithotrophicus ES-1 and homologs in Leptospirillum spp. further suggests that they may have a common function in electron transport under microaerophilic conditions and possibly have a role in iron oxidation. Although the specific function of these genes remains unclear, their conservation among the Zetaproteobacteria and other iron oxidizers indicates that they warrant further investigation.

\section{Oxidative stress and protection}

Production of reactive $\mathrm{O}_{2}$ species such as superoxide and hydroxyl radicals can occur under the high $\mathrm{Fe}(\mathrm{II})$ and fluctuating $\mathrm{O}_{2}$ conditions (Cabiscol et al., 2010) that the Zetaproteobacteria are exposed to. Compared with other SAGs, members of OTU 10 appear to have greater protection against reactive $\mathrm{O}_{2}$ species. Superoxide dismutase is largely absent in the SAGs being present only in SAGs from OTU 10 and the isolates. There are also only two SAGs that encode catalase genes, one of which is OTU 10 SAG AC-673-M07 (Supplementary Table S13). Additionally, two OTU 10 members have bacterioferritin, which is absent in all other Zetaproteobacteria genomes. Bacterioferritin is intriguing as it has been shown to be involved in both iron storage and protection against oxidative damage created by the reaction of ferrous iron with $\mathrm{O}_{2}$ (Carrondo, 2003). This increased repertoire of genes involved in mitigating oxidative stress suggests that OTU 10 may be adapted to fluctuating $\mathrm{O}_{2}$ conditions compared with other uncultivated Zetaproteobacteria. It is currently unclear whether the depth within the mat, geographical location, or other factors have a role in the development of these attributes and should be evaluated further. 
Nitrogen metabolism

Previous studies have indicated that Loihi Seamount vent fluids are enriched in ammonium and depleted in nitrate and nitrite compared with ambient seawater (Sedwick et al., 1992; Karl et al., 1988; Garcia et al., 2006). Strains PV-1 and M34 were both isolated using medium in which ammonium was the sole nitrogen source, and genomic analysis indicates that they, as well as 13 SAGs, encode the genes necessary to utilize ammonium (Figure 7 and Supplementary Table S14). In addition, 11 SAGs have nitrate reductases, all of which show structural similarities to assimilatory nitrate reductases (Moreno-Vivián et al., 1999; Kraft et al., 2011). Nine SAGs contain nitrite reductases (both large and small subunits). This indicates that almost half of the analyzed cells have the potential to assimilate nitrate. These same SAGs also contain nitrate/nitrite transporters as well as the nitrogen assimilation regulatory genes $n t r B / n t r C$ (LuqueAlmagro et al., 2011) directly upstream. Strain M34 encodes nitrogenase genes, although their functionality remains to be verified. Nitrogenase genes were surprisingly absent in all of the SAGs. Eleven SAGs as well as both isolates have nitrilase genes, which have been found to hydrolyze organic nitrogen compounds and produce ammonia (Podar et al., 2005). One SAG (AB-137_G06) also contains a nitronate monooxygenase gene that may be involved in carbon-nitrogen compound degradation through the use of $\mathrm{O}_{2}$ and forming nitrite as one of its degradation products (Gadda and Francis, 2010). There is also genetic evidence for potential urea utilization through the presence of transporters and urea carboxylase in both strains PV-1 and M34, as well as a urease gene in one of the SAGs (AC-673-M07).

There appears to be niche specialization among the Zetaproteobacteria in the type of nitrogen source

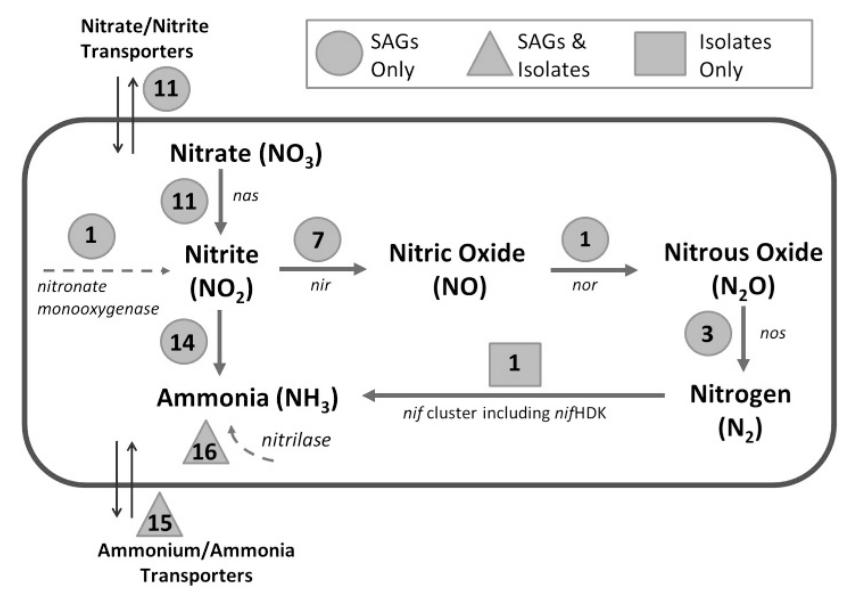

Figure 7 Potential nitrogen transformations by Zetaproteobacteria based on genomic analysis of 23 SAGs and two sequenced isolates (M. ferrooxydans PV-1 and $M$. ferrooxydans M34). Symbols represent in which genomic source genes were found. Numbers within symbols represent the number of genomes in which that gene was found. Broken lines denote unknown source compounds. they can use, whether it is nitrate, ammonium, organic compounds, nitrogen gas or some intermediate. Most have the potential to take up ammonium, but it appears as though some have acquired additional mechanisms for obtaining nitrogen. The partial recovery of genomes does not allow us to speculate on completeness of pathways as some of the genes may not have been recovered, but overall the detection of these genes in a high proportion of the SAGs suggest that they may have functional importance in the Zetaproteobacteria. Nitrogen availability, competition between cells, or fluctuating $\mathrm{O}_{2}$ conditions may lead to the switch between nitrogen sources. It is surprising to find nitrate assimilation genes in so many of the SAGs, as the nitrate concentrations previously measured in the vent fluids were extremely low. The source of nitrate remains unknown, but it is possible that it comes from ambient seawater or that there are ammonia-oxidizing or other nitrifying microbes within the mats that are producing nitrate. The Zetaproteobacteria may also compete with these organisms for ammonia or $\mathrm{O}_{2}$ leading to the development of these alternative nitrogen pathways. It appears as though Zetaproteobacteria use nitrate directly for a nitrogen source, although it cannot be ruled out that nitrate may be used as an alternative electron acceptor under certain conditions. Although there are a number of nitrate-dependent iron-oxidizing bacteria that grow under anaerobic conditions (Weber et al., 2006), thus far there is no direct evidence that the Zetaproteobacteria are capable of this metabolism. These results indicate that the Zetaproteobacteria may have more diverse systems for acquiring nitrogen and therefore have a more significant role in nitrogen transformations than previously believed. The interactions between the Zetaproteobacteria living within the mats and different forms of nitrogen may be complex, dynamic, and represent a significantly understudied process in these environments.

Using genomic insights to isolate novel Zetaproteobacteria The results presented here pose new enrichment strategies through which novel Zetaproteobacteria may be isolated. Enrichments targeting a wider range of $\mathrm{O}_{2}$ conditions may lead to the isolation of some of these higher and lower $\mathrm{O}_{2}$-tolerant Zetaproteobacteria. For example, higher $\mathrm{O}_{2}$-tolerant Zetaproteobacteria appear to be largely from OTU 10, which are more prevalent at Marker 57 and could be targeted from this site in future sampling efforts. Similarly, they were also found in both bulk mat samples, suggesting that suction samples may be more appropriate starting material for inoculating enrichments specific for these organisms. The use of a variety of available nitrogen sources as well as the addition of heterotrophic or complex carbon sources may be advantageous for isolating some of these novel groups as well. 
Although we cannot demonstrate that the genomes obtained for this study are from cells that are carrying out iron oxidation, there is surprisingly little genomic evidence in these 23 SAGs of other lithotrophic metabolisms, including sulfur oxidation, ammonia oxidation, and methane oxidation. The possible exception being hydrogen oxidation because of the presence of Type I and Type III Ni-Fe hydrogenases and cytochrome b561 genes that have been implicated in hydrogen oxidation processes (Yoon et al., 2008) (Supplementary Table S15). Another finding gleaned from the SAGs is the presence of aerobic and anaerobic cobalamin (vitamin $\mathrm{b}_{12}$ ) biosynthesis genes and corresponding cobalt transporters (Supplementary Tables S16 and S17), indicating that enrichments lacking B vitamins may be a relatively easy way to enrich novel strains.

We used the data presented here to isolate Mariprofundus sp. EKF-M39, a novel stalk-forming, iron-oxidizing Zetaproteobacteria strain from Loihi Seamount using medium containing nitrate as the sole nitrogen source, under very low $\mathrm{O}_{2}$ conditions. Based on 16S rRNA gene comparisons, this isolate is most closely related to strain PV-1 and strain M34, but at only 96\% gene similarity it is the most phylogenetically distinct Zetaproteobacteria isolate obtained from Loihi Seamount to date (Supplementary Figure S5). The genome of strain EKF-M39 was sequenced (2.7 Mb) and whole genome comparisons indicate that it is even more distant from these two isolates at the ANI and AAI levels (71\% and $69 \%$, respectively). Compared with the SAGs, this isolate has an average similarity of $93 \%$ based on $16 \mathrm{~S}, 67 \%$ ANI and $59 \%$ AAI.

Physiologic studies with strain EKF-M39 were conducted in an effort to verify some of the predicted SAG functions. This organism carries out lithotrophic iron oxidation under microaerophilic conditions. Strain EKF-M39 has phosphotransferase system genes, but did not show evidence for heterotrophic growth on mannose, fructose, acetate, or cellobiose when these were provided as the sole carbon sources (Table 2 and Supplementary Methods). It also is unable to carry out vitamin $\mathrm{B}_{12}$ auxotrophy under iron-oxidizing conditions or grow with hydrogen as the electron donor. Most interestingly, strain EKF-M39 confirms that the Zetaproteobacteria are versatile in their nitrogen utilization capabilities as this strain can grow using either nitrate or ammonium as the sole nitrogen source. It can also grow in nitrogen-deplete medium, which is consistent with nitrogen fixation. Strain EKF-M39 has the genetic capacity to carry out different modes of nitrogen assimilation and has a full suite of nitrogen fixation genes confirming the physiologic tests. Strain EKF-M39 is not capable of anaerobic nitrate-dependent iron oxidation (Table 2 and Supplementary Methods), confirming that it uses nitrate solely as a nitrogen source.

The genomic potential of this isolate supports additional findings introduced by the analysis of the SAGs. It represents the first Zetaproteobacteria isolate to contain a Cluster 1 molybdopterin oxidoreductase gene (Supplementary Figure S6) and lack the Cluster 2 molybdopterin oxidoreductase. The Cluster 1 molybdopterin oxidoreductase gene and corresponding operon in strain EKF-M39 are the only nitrate assimilation genes in the near-complete genome, indicating that these are likely responsible for the growth by strain EKF-M39 when nitrate was the sole nitrogen source. This possibly indicates that strain EKF-M39 has a different mechanisms for iron oxidation compared with strains PV-1 and M34, and/or the Cluster 2 molybdopterin oxidoreductase genes are not essential for iron oxidation. Strain EKF-M39 also has the conserved set of cytochrome genes found in strains PV-1, M34, and S. lithotrophicus ES-1, supporting the potential importance of this gene operon in electron transport and possibly in iron oxidation. Last, it is the first Zetaproteobacteria isolate to have only a form II RubisCO gene (Supplementary Figure S7), which is consistent with the very low $\mathrm{O}_{2}$ enrichment conditions used to isolate this organism, demonstrating that this strategy can be successful for finding unique isolates. Additional in-depth physiological and genomic analyses of this isolate are currently being conducted and will be described elsewhere.

Table 2 Physiological characteristics of the iron-oxidizing isolate Mariprofundus sp. EKF-M39

\begin{tabular}{|c|c|c|c|}
\hline Oxygen conditions & Type of growth & Substrate & Growth \\
\hline \multirow[t]{11}{*}{ Microaerophilic } & Autotrophy & $\mathrm{CO}_{2}$ & Yes \\
\hline & Heterotrophy & Mannose & No \\
\hline & & Fructose & No \\
\hline & & Sodium acetate & No \\
\hline & & Cellobiose & No \\
\hline & Nitrogen utilization & Ammonium chloride & Yes \\
\hline & & Sodium nitrate & Yes \\
\hline & & $\mathrm{N}$-deplete $\left(\mathrm{N}_{2}\right.$ only) & Yes \\
\hline & Vitamin $\mathrm{B}_{12}$ auxotrophy & No vitamins & No \\
\hline & & Riboflavin + Vit $B_{5}$ only & No \\
\hline & Hydrogen oxidation & $\mathrm{H}_{2}$ & No \\
\hline Anaerobic & Nitrate-dependent iron oxidation & Sodium nitrate & No \\
\hline
\end{tabular}




\section{Conclusions}

Partial genome sequences from 23 uncultivated Zetaproteobacteria from Loihi Seamount significantly expand our understanding of the genetic diversity, metabolic potential, and ecologic roles of this elusive taxonomic group. This study suggests that $\mathrm{O}_{2}$ tolerances and nitrogen metabolisms may have a significant role in niche specialization among the Zetaproteobacteria. Future analyses of fine-scale sampling efforts within these mats will be necessary to better understand the extent of these niche differentiations. Aside from Fe-oxidation, there was a surprising lack of other lithotrophic or anaerobic metabolisms in the Zetaproteobacteria SAGs at Loihi Seamount. This may explain why previous studies have identified these organisms almost exclusively in high iron environments. Genomic comparisons with Zetaproteobacteria from non-hydrothermal vent sites and other geographic locations will also help determine the true breadth of their metabolic versatility on a global scale. Overall, these findings lay the groundwork for future laboratory and in situ studies as they provide insight into the potential lifestyle of the Zetaproteobacteria.

\section{Conflict of Interest}

The authors declare no conflict of interest.

\section{Acknowledgements}

We thank Brian Thompson, Nicole Poulton, and Elizabeth Fergusson for their technical expertise and support in single-cell sorting and sequencing, as well as Anna Leavitt for her assistance with the physiologic studies. We also thank the captains, crew and operators of the R/V Kilo Moana, R/V Thomas N Thompson, and Jason II ROV without whom this work would not have been possible. Last, we appreciate the intellectual input provided by the members of the Single Cell Genomics Center and Geomicrobiology labs at Bigelow. This work was financially supported by the NASA EPSCoR program administered through the Maine Space Consortium Grant 10-EPSCoR0005 and the National Science Foundation Grants OCE0821374 and OCE-1155754. The work conducted by the US Department of Energy Joint Genome Institute is supported by the Office of Science of the US Department of Energy under Contract No. DE-AC02-05CH11231. Additional sequencing support for Bigelow Laboratory was provided through the Joint Genome Institute's Community Sequencing Program, Project 560.

\section{References}

Amouric A, Brochier-Armanet C, Johnson DB, Bonnefoy V, Hallberg KB. (2011). Phylogenetic and genetic variation among Fe(II)-oxidizing acidithiobacilli supports the view that these comprise multiple species with different ferrous iron oxidation pathways. Microbiology 157: $111-122$.
Badger MR, Bek EJ. (2008). Multiple Rubisco forms in proteobacteria: their functional significance in relation to $\mathrm{CO}_{2}$ acquisition by the CBB cycle. J Exp Bot 59: 1525-1541.

Berg IA. (2011). Ecological aspects of the distribution of different autotrophic $\mathrm{CO}_{2}$ fixation pathways. Appl Environ Microbiol 77: 1925-1936.

Borisov VB, Gennis RB, Hemp J, Verkhovsky MI. (2011). The cytochrome bd respiratory oxygen reductases. BBA-Bioenergetics 1807: 1398-1413.

Cabiscol E, Tamarit J, Ros J. (2010). Oxidative stress in bacteria and protein damage by reactive oxygen species. Int Microbiol 3: 3-8.

Carrondo MA. (2003). Ferritins, iron uptake and storage from the bacterioferritin viewpoint. EMBO J 22: 1959-1968.

Chan CS, Fakra SC, Emerson D, Fleming EJ, Edwards KJ. (2011). Lithotrophic iron-oxidizing bacteria produce organic stalks to control mineral growth: implications for biosignature formation. ISME J 5: 717-727.

Cox JC, Boxer DH. (1978). The purification and some properties of rusticyanin, a blue copper protein involved in iron(II) oxidation from Thiobacillus ferro-oxidans. Biochem J 174: 497-502.

Croal LR, Jiao Y, Newman DK. (2007). The fox operon from Rhodobacter strain SW2 promotes phototrophic Fe(II) oxidation in Rhodobacter capsulatus SB1003. J Bacteriol 189: 1774-1782.

Dang H, Chen R, Wang L, Shao S, Dai L, Ye Y et al. (2011). Molecular characterization of putative biocorroding microbiota with a novel niche detection of Epsilonand Zetaproteobacteria in Pacific Ocean coastal seawaters. Environ Microbiol 13: 3059-3074.

Deutscher J, Aké FMD, Derkaoui M, Zébré AC, Cao TN, Bouraoui H et al. (2014). The bacterial phosphoenolpyruvate: carbohydrate phosphotransferase system: regulation by protein phosphorylation and phosphorylation-dependent protein-protein interactions. Microbiol Mol Biol Rev 78: 231-256.

Emerson D, Floyd MM. (2005). Enrichment and isolation of iron-oxidizing bacteria at neutral pH. Methods Enzymol 397: 112-123.

Emerson D, Moyer CL. (2010). Microbiology of seamounts: common patterns observed in community structure. Oceanography 23: 148-163.

Emerson D, Field EK, Chertkov O, Davenport KW, Goodwin L, Munk C et al. (2013). Comparative genomics of freshwater Fe-oxidizing bacteria: implications for physiology, ecology, and systematics. Front Microbiol 4: 254.

Fleming EJ, Davis RE, McAllister SM, Chan CS, Moyer CL, Tebo BM et al. (2013). Hidden in plain sight: discovery of sheath-forming, iron-oxidizing Zetaproteobacteria at Loihi Seamount, Hawaii, USA. FEMS Microbiol Ecol 85: 116-127.

Gadda G, Francis K. (2010). Nitronate monooxygenase, a model for anionic flavin semiquinone intermediates in oxidative catalysis. Arch Biochem Biophys 493: 53-61.

Garcia MO, Caplan-Auerbach J, De Carlo EH, Kurz MD, Becker N. (2006). Geology, geochemistry and earthquake history of Loihi Seamount, Hawaìi's youngest volcano. Chem Erde-Geochem 66: 81-108.

Garcia SL, McMahon KD, Martinez-Garcia M, Srivastava A, Sczyrba A, Stepanauskas $\mathrm{R}$ et al. (2012). Metabolic potential of a single cell belonging to one of the most abundant lineages in freshwater bacterioplankton. ISME J 7: 137-147. 
Glazer BT, Rouxel OJ. (2009). Redox speciation and distribution within diverse iron-dominated microbial habitats at Loihi Seamount. Geomicrobiol $J$ 26: $606-622$.

Jiao Y, Newman DK. (2007). The pio operon is essential for phototrophic Fe(II) oxidation in Rhodopseudomonas palustris TIE-1. J Bacteriol 189: 1765-1773.

Kamke J, Sczyrba A, Ivanova N, Schwientek P, Rinke C, Mavromatis K et al. (2013). Single-cell genomics reveals complex carbohydrate degradation patterns in poribacterial symbionts of marine sponges. ISME J 7: 2287-2300.

Karl D, McMurtry G, Malahoff A, Garcia M. (1988). Loihi Seamount, Hawaii: a mid-plate volcano with a distinctive hydrothermal system. Nature 335: $532-535$

Katoh K, Misawa K, Ki Kuma, Miyata T. (2002). MAFFT: a novel method for rapid multiple sequence alignment based on fast Fourier transform. Nucleic Acids Res 30: 3059-3066.

Konstantinidis KT, Tiedje JM. (2005). Towards a genomebased taxonomy for prokaryotes. J Bacteriol 187: 6258-6264.

Konstantinidis KT, Ramette A, Tiedje JM. (2006). The bacterial species definition in the genomic era. Philos Trans Roy Soc Ser B 361: 1929-1940.

Kraft B, Strous M, Tegetmeyer HE. (2011). Microbial nitrate respiration-genes, enzymes and environmental distribution. J Biotechnol 155: 104-117.

Kumar S, Nei M, Dudley J, Tamura K. (2008). MEGA: a biologist-centric software for evolutionary analysis of DNA and protein sequences. Brief Bioinform 9: 299-306.

Lasken RS, Stockwell TB. (2007). Mechanism of chimera formation during the Multiple Displacement Amplification reaction. BMC Biotechnol 7: 19.

Ludwig W, Strunk O, Westram R, Richter L, Meier H. (2004). ARB: a software environment for sequence data. Nucleic Acids Res 32: 1363-1371.

Luque-Almagro VM, Gates AJ, Moreno-Vivián C, Ferguson SJ, Richardson DJ, Roldán M. (2011). Bacterial nitrate assimilation: gene distribution and regulation. Biochem Soc T 39: 1838-1843.

McAllister SM, Davis RE, McBeth JM, Tebo BM, Emerson D, Moyer CL. (2011). Biodiversity and emerging biogeography of the neutrophilic ironoxidizing Zetaproteobacteria. Appl Environ Microbiol 77: 5445-5457.

McBeth JM, Little BJ, Ray RI, Farrar KM, Emerson D. (2011). Neutrophilic iron-oxidizing 'Zetaproteobacteria' and mild steel corrosion in nearshore marine environments. Appl Environ Microbiol 77: 1405-1412.

Moreno-Vivián C, Cabello P, Martínez-Luque M, Blasco R, Castillo F. (1999). Prokaryotic nitrate reduction: molecular properties and functional distinction among bacterial nitrate reductases. J Bacteriol 181: 6573-6584.

Pitcher RS, Watmough NJ. (2004). The bacterial cytochrome $\mathrm{Cbb}_{3}$ oxidases. Biochim Biophys Acta Bioenerget 1655: 388-399.

Podar M, Eads JR, Richardson TH. (2005). Evolution of a microbial nitrilase gene family: a comparative and environmental genomics study. BMC Evol Biol 5: 42 .

Pruesse E, Quast C, Knittel K, Fuchs BM, Ludwig W, Peplies J et al. (2007). SILVA: a comprehensive online resource for quality checked and aligned ribosomal
RNA sequence data compatible with ARB. Nucleic Acids Res 35: 7188-7196.

Rassa AC, McAllister SM, Safran SA, Moyer CL. (2009). Zeta-proteobacteria dominate the colonization and formation of microbial mats in low-temperature hydrothermal vents at Loihi Seamount, Hawaii. Geomicrobiol J 26: 623-638.

Richter M, Rosselló-Móra R. (2009). Shifting the genomic gold standard for the prokaryotic species definition. Proc Natl Acad Sci USA 106: 19126-19131.

Rinke C, Schwientek P, Sczyrba A, Ivanova NN, Anderson IJ, Cheng J-F et al. (2013). Insights into the phylogeny and coding potential of microbial dark matter. Nature 499: 431-437.

Schloss P, Handelsman J. (2005). Introducing DOTUR, a computer program for defining operational taxonomic units and estimating species richness. Appl Environ Microbiol 71: 1501-1506.

Sedwick PN, McMurtry G, Macdougall J. (1992). Chemistry of hydrothermal solutions from Pele's vents, Loihi Seamount, Hawaii. Geochim Cosmochim Acta 56: 3643-3667.

Shi L, Rosso KM, Clarke TA, Richardson DJ, Zachara JM, Fredrickson JK. (2012). Molecular underpinnings of Fe(III) oxide reduction by Shewanella oneidensis MR-1. Front Microbiol 3: 50.

Singer E, Emerson D, Webb EA, Barco RA, Kuenen JG, Nelson WC et al. (2011). Mariprofundus ferrooxydans PV-1 the first genome of a marine Fe(II) oxidizing Zetaproteobacterium. PLoS One 6: e25386.

Singer E, Heidelberg JF, Dhillon A, Edwards KJ. (2013). Metagenomic insights into the dominant Fe(II) oxidizing Zetaproteobacteria from Lo'ihi, Hawai'I. Front Microbiol 4: 52.

Singer SW, Chan CS, Zemla A, VerBerkmoes NC, Hwang M, Hettich RL et al. (2008). Characterization of cytochrome 579, an unusual cytochrome isolated from an iron-oxidizing microbial community. Appl Environ Microbiol 74: 4454-4462.

Staley JT. (2006). The bacterial species dilemma and the genomic-phylogenetic species concept. Philos Trans $R$ Soc Ser B 361: 1899-1909.

Stamatakis A. (2006). RAxML-VI-HPC: maximum likelihood-based phylogenetic analyses with thousands of taxa and mixed models. Bioinformatics 22: 2688-2690.

Stepanauskas R, Sieracki ME. (2007). Matching phylogeny and metabolism in the uncultured marine bacteria, one cell at a time. Proc Natl Acad Sci USA 104: 9052-9057.

Swan BK, Martinez-Garcia M, Preston CM, Sczyrba A, Woyke T, Lamy D et al. (2011). Potential for chemolithoautotrophy among ubiquitous bacteria lineages in the dark ocean. Science 333: 1296-1300.

Swan BK, Tupper B, Sczyrba A, Lauro FM, MartinezGarcia M, González JM et al. (2013). Prevalent genome streamlining and latitudinal divergence of planktonic bacteria in the surface ocean. Proc Natl Acad Sci USA 110: 11463-11468.

Tabita FR, Satagopan S, Hanson TE, Kreel NE, Scott SS. (2008). Distinct form I, II, III, and IV Rubisco proteins from the three kingdoms of life provide clues about Rubisco evolution and structure/function relationships. J Exp Bot 59: 1515-1524.

Thompson JD, Higgins DG, Gibson TJ. (1994). CLUSTAL W: improving the sensitivity of progressive multiple sequence alignment through sequence weighting, 
position-specific gap penalties and weight matrix choice. Nucleic Acids Res 22: 4673-4680.

Weber KA, Achenbach LA, Coates JD. (2006). Microorganisms pumping iron: anaerobic microbial iron oxidation and reduction. Nat Rev Microbiol 4: 752-764.

Wilkins MJ, Kennedy DW, Castelle CS, Field EK, Stepanauskas R, Fredrickson JK et al. (2014). Singlecell genomics reveal metabolic strategies for growth and survival in an oligotrophic aquifer. Microbiology 160: $362-372$
Woyke T, Xie G, Copeland A, Gonzalez JM, Han C, Kiss H et al. (2009). Assembling the marine metagenome, one cell at a time. PLoS One 4: e5299.

Woyke T, Sczyrba A, Lee J, Rinke C, Tighe D, Clingenpeel S et al. (2011). Decontamination of MDA reagents for single cell whole genome amplification. PLoS One 6: e26161.

Yoon KS, Tsukada N, Sakai Y, Ishii M, Igarashi Y, Nishihara H. (2008). Isolation and characterization of a new facultatively autotrophic hydrogen-oxidizing Betaproteobacterium, Hydrogenophaga sp. AH-24. FEMS Microbiol Lett 278: 94-100.

Supplementary Information accompanies this paper on The ISME Journal website (http://www.nature.com/ismej) 